\title{
Immobilization of hydrophobic lipase derivatives on to organic polymer beads
}

\begin{abstract}
A simple and effective method of lipase immobilization is described. Lipase from Candida rugosa was first modified with several hydrophobic modifiers before being adsorbed on to organic polymer beads. The soluble hydrophobic lipase derivatives adsorbed more strongly on to the various polymers as compared with the native lipase. The optimal adsorption temperature of the native and modified lipases on all the polymers was $40^{\circ} \mathrm{C}$. The optimal $\mathrm{pH}$ of adsorption was between 6 and 7. Lipase immobilized in this manner produced high catalytic recoveries which were affected by the type of modifiers, degree of modification and type of supports used. Monomethoxypolyethylene glycol (1900) activated with p-nitrophenyl chloroformate was found to be the best modifier of the enzyme at $95 \%$ modification, for adsorption to the polymers. Increasing the degree of modification of the enzyme increased the activity which was immobilized. Generally, both native and hydrophobic lipase derivatives showed higher specific activities when immobilized on polar polymers compared with nonpolar polymers.
\end{abstract}

Keyword: Lipase; Hydrophobic modifiers; Modification; Immobilization; Immobilized activity 